Original Research Paper

\title{
On the Sensitivity of a Dynamic Measure of Financial Inequality
}

\author{
${ }^{1}$ Guglielmo D'Amico, ${ }^{2}$ Stefania Scocchera and ${ }^{1}$ Loriano Storchi \\ ${ }^{I}$ Department of Pharmacy, University G. D'Annunzio, Chieti, Italy \\ ${ }^{2}$ Department of Management, University Politecnica Delle Marche, Ancona, Italy
}

\author{
Article history \\ Received: 29-06-2019 \\ Revised: $12-09-2019$ \\ Accepted: 24-10-2019 \\ Corresponding Author: \\ Guglielmo D'Amico \\ Department of Pharmacy, \\ University G. D’Annunzio, \\ Chieti, Italy \\ Email: g.damico@unich.it
}

\begin{abstract}
In the present work, we investigate the sensitivity of the dynamic Theil index computed under a Markov reward model with respect to structured perturbations affecting the underlying Markov process. The model is applied to the sovereign credit spread evolution as a proxy for financial risk, which are driven by the sovereign credit rating dynamic. The introduction of such perturbations allows to evaluate the sensitivity of the inequality of the financial risk in a given group of financial entities with respect to the uncertainty in the rating dynamics. To this end we perform a simulation based sensitivity analysis. The methodology is applied to real data concerning sovereign credit ratings and long-term interest rates on government bonds of 24 European countries. Obtained results suggest different sensitivity of the inequality measure to the 12 scenarios built supposing different ways the perturbations could affect the rating process.
\end{abstract}

Keywords: Dynamic Inequality, Credit Rating, Credit Spread, Sensitivity Analysis

\section{Introduction}

Sovereign credit risk has become of main interest during the last decades due to the occurrence of events such as economic and financial crisis which have affected the sovereign default probability. The implication of a country's default and generally, of an increase of the sovereign credit risk is of crucial importance concerning both its effects on the domestic economy but also on the neighborhood economies. We have focused our attention on the financial risk in European Union by applying dynamic measure of inequality to evaluate how the financial risk is distributed among countries and to assess its behavior over time (D'Amico et al., 2018a; 2018b; 2019). The financial risk of a country refers to the ability to cope with its financial commitments and it is expressed by the amount of credit spread that the country has to pay on its debt. The inequality measure gives us information about the degree of dispersion of this risk among the countries. Thus, it can be interpreted as a measure of risk when related to a group of countries or generally, of financial entities. More specifically, we assume that the evolution of the credit spreads is influenced by the credit ratings, which evolve according to a Continuous-Time Markov Chain (CTMC). In the present work, we are interested in gaining more insight on the influence played by rating dynamics on the evolution of the inequality process and on the sensitivity of the measure of financial risk when estimation errors occur. Thus, starting from our previous work, (D'Amico et al., 2018b), a sensitivity analysis is carried out by adding a perturbation to the generator of the CTMC, in such a way to be able to depict the possible evolution of the inequality considering the uncertainty in the specification of the generator of the Markov process. The introduction of a perturbation for Markov process has been studied in several works. The sensitivity of steady state solution of Markov chain has been investigated, among others, in (Schweitzer, 1968) and successively a study of the sensitivity bounds for stationary distribution of Markov chain has been presented in (Haviv and Van der Heyden, 1984). Ramesh and Trivedi (1993) the authors derive measure of sensitivity and its bounds for transient solutions under structured and unstructured perturbations for CTMC. The study of the effect of perturbations for a discrete-time Markov reward process on the total expected reward has, instead, been performed in (Yu et al., 2009). Do Van et al. (2010) a structured perturbation for Markov reliability models has been proposed for application of decision-making. Although Markov processes have been widely applied in financial literature (see among others, (Doubleday and Esunge, 2011; Thyagarajan and Saiful, 2005; Trueck and Rachev, 2009)), the Markovian property has been criticized in several works, especially related to credit 
rating dynamics. Firstly, Carty and Fons (1994) showed that rating life span is described by Weibull distribution. Successively, the use of semi-Markov chains as a more suitable model to describe rating dynamics has been proposed by (D'Amico et al., 2006; 2017). However, in our application, we cannot apply semi-Markov processes because of the sparsity of the sovereign rating data. We instead rely on the continuous time homogeneous Markov chain to model the rating dynamics. The advantages of working within a continuous-time framework are well known and documented in literature (Christensen et al., 2004; Lando and Skødeberg, 2002; Jarrow et al., 1997) and for the financial problem we posed (D'Amico et al., 2018b). Applications of information theory for economic and financial problems have been advanced in (D'Amico and Regnault, 2018) where the authors proposed a dynamic extension of common poverty indices. D'Amico et al. (2012) a dynamic measurement of income inequality based on the Theil index has been studied. Successively a decomposition of this measure has been advanced in (D'Amico et al., 2014). The measure of inequality we apply in order to evaluate the financial risk is based on the last two contributions. To the best of our knowledge, a sensitivity analysis of the dynamic inequality with respect to the uncertainty in the rating dynamics has never been faced. Following (Ramesh and Trivedi, 1993), we introduce a structured perturbation and we perform several simulations by varying the perturbation parameters and the way they affect the generator of the CTMC. This is done to analyze the differences on the measure of inequality assessed in the nominal and the perturbed model (i.e., that one without perturbation and that one with the introduction of a perturbation, respectively). The methodology is applied to real data concerning sovereign credit rating data from Standard \& Poor's (S\&P) and interest rates of long-term government bonds for 24 European countries. Regarding the values of the perturbations, they are generated according to Normal distributions and from multivariate Normal distributions to include dependence of perturbations among rating classes. This is done by supposing three cases where the perturbation affects all off-diagonal intensities, the downgrade intensities and the upgrade intensities of the generator matrix, respectively. The simulations highlight the importance of including the dependence among rating classes while introducing a perturbation parameter as the results are sensitive to variations in the covariance values. Furthermore, the results suggest also different sensitivity of the inequality measure when perturbations affect differently the exit rates of the generator, as there are differences in the three cases exposed above. The paper is organized as follows: Section 1 gives an overview of the data collected while section 2 describes the model. In Section 3 we introduce the dynamic entropy under the nominal model and the measure for the sensitivity with respect to structured perturbations. The fourth Section describes the way the simulations are carried out and the results of these simulations by building 12 scenarios where the perturbations differently affect the rating dynamics. Finally in the last Section, concluding remarks are given.

\section{Data}

Our objective is to provide a model able to measure the financial inequality in a set of countries and the role that some perturbations could play in the variation of this measure. To this end, we focused on European countries and we collected data on two main variables: The sovereign credit ratings and the credit spreads. Sovereign credit rating is an ordinal measure of the country's credit risk. It expresses the ability of a country to face its financial commitments. Sovereign credit spreads are the difference between interest rates of various countries and they represent a proxy for the financial risk related to a specific country. The sovereign credit ratings assigned to the European countries by Standard and Poor's have been collected from November 23, 1998 to June 26, 2018 on a daily scale, from the Tradingeconomics website. In this way, the resulting dataset describes the evolution of the creditworthiness (expressed by the rating) of the European countries over the last two decades. The rating agencies have established letters system to describe the creditworthiness of the issuers, which is composed by a large number of rankings. As usually done in the literature, we group all these rankings into eight rating classes: $\mathrm{AAA}, \mathrm{AA}, \mathrm{A}, \mathrm{BBB}, \mathrm{BB}, \mathrm{B}, \mathrm{C}$, $\mathrm{D}$ where $\mathrm{C}=\{C C C, C C, C\}$, that we will denotes as $1,2, \ldots, 8$ for seek of simplicity. Rating class AAA $=1$ denotes that the issuer has the capability to easily face with its financial commitments, whereas lower credit ratings (i.e., from 2 to 7 ) denote that the issuer is gradually less able to cope with its commitments. The last class indicates both Selected Default and Default. Commonly the first four rating class (AAA, AA, A, $\mathrm{BBB})$ are known as Investment-grade rating classes while the others (BB-B-C-D) as speculative-grade rating classes. The former are assigned to issuers with relatively low to moderate default risk. The latter are instead assigned to obligors with an higher default risk until the event of default. During the observed period only 5 countries experienced a downgrade to a speculative rating class (Bulgaria, Croatia, Greece, Portugal and Romania). Most of them were assigned starting from 2012. Figure 1 shows the observed rating trajectories for Greece, Italy, Hungary and Portugal over the period ranging between November 1998 and June 2018 (the time-line is shown in the horizontal axis, while the vertical axis represents the rating class). While Italian rank gradually deteriorates (from AA to BBB) with two downgrades, Hungary and Portugal experienced a different assignment with 4 transitions. The first one initially ameliorates its creditworthiness (from BBB to 
A), during the financial crisis it came back to rating BBB until it deteriorated to a speculative rating class (BB) up to the 2016 when there was an upgrade to rank $\mathrm{BBB}$. On the contrary, the Portugal assignment has been stable (AA) until the financial crisis. After that period, Portugal experienced a quite rapid deterioration up to rating BB. Finally, Greece has had 11 transitions, most of them concentrated around the 2012 when Selected Default has been assigned to Greece two times. The second variable collected is the credit
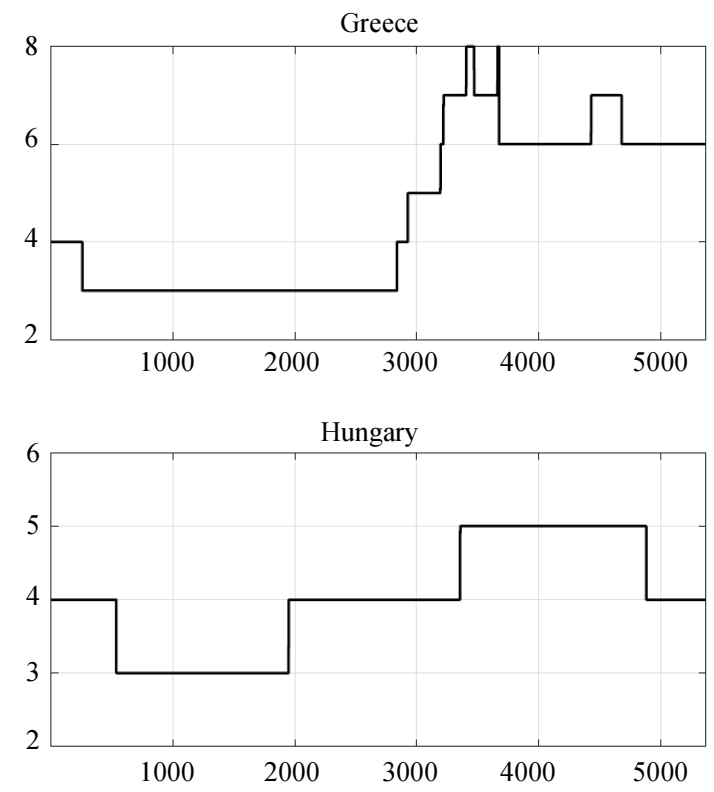

spread, which is given by the difference between the interest rate and a benchmark. In our case, the benchmark is the minimum value among the interest rates paid by all European countries. The reason is that there are some countries experiencing lower interest rates than Germany. Therefore, the long-term interest rate of sovereign government bonds with 10 year maturity are collected from the investing.com web-site, on a daily scale. The data are available for all countries starting from April, 26, 2010.
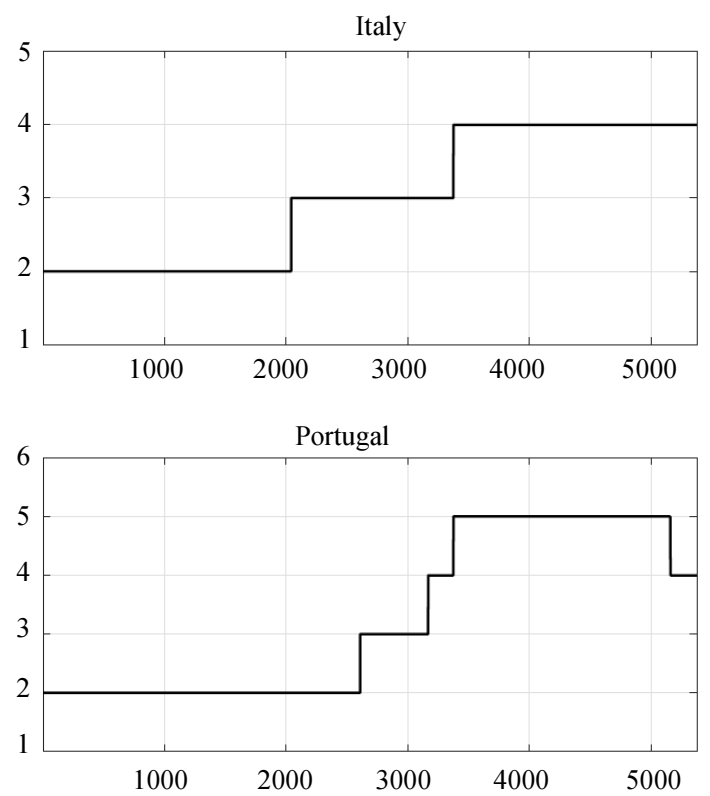

Fig. 1: Rating assignments for Greece, Italy; Hungary and Portugal
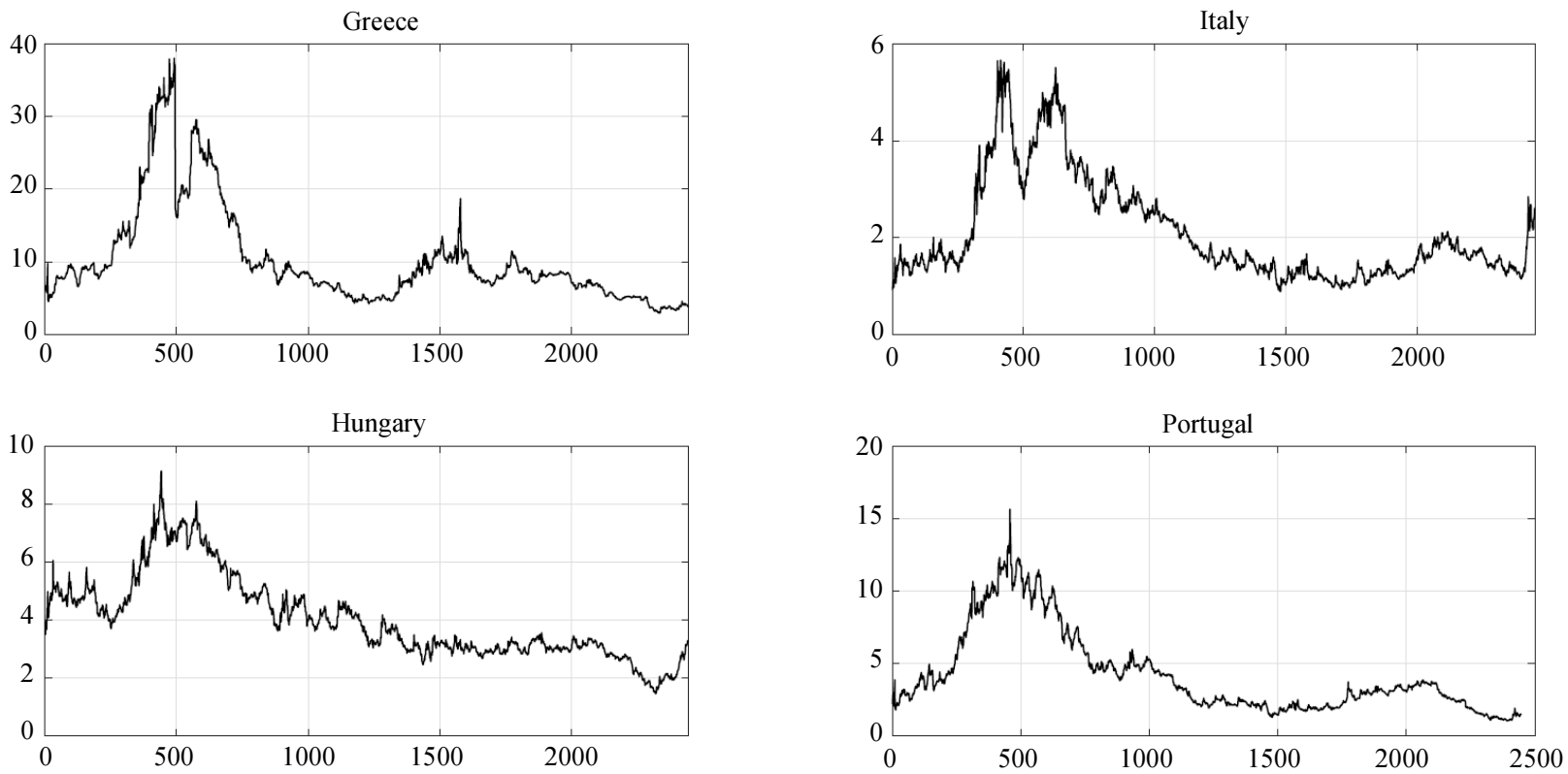

Fig. 2: Credit spreads (\%) paid by Greece, Italy; Hungary and Portugal 
Thus, the observation period ranges between this date and June 26, 2018. Figure 2 illustrates the credit spread paid by Greece, Italy, Hungary and Portugal over the observed period. The credit spread is in percentage value (in the vertical axis). The countries' payments shows almost a similar trend with two peaks between 2012 and 2013 which are more pronounced for the first two countries (except for Portugal for whom there is only one peak). However, the spread values are different: Greece has paid the highest credit spread reaching 38\%, followed by Portugal, Hungary and Italy. In particular, Greece has paid the highest spread of all sample and the other countries' payments have shown similar trend to those of Hungary and Italy. After the 2013 the spreads shown a decreasing trend except for Greece which has registered an other increase around the 2016.

As not all data were available for all European countries the sample is composed by 24 members: Belgium, Bulgaria, Czech Republic, Germany, Denmark, Ireland, Greece, Spain, France, Croatia, Italy, Lithuania, Hungary, Malta, Netherlands, Austria, Poland, Portugal, Romania, Slovenia, Slovakia, Finland, Sweden, United Kingdom.

\section{The Model}

The model we are going to present in this section is in line with that considered in (D'Amico and Regnault, 2018) related to a different problem.

Let $C$ be a group of $N$ countries joining the Euro area. Each country $c \in C$ is ranked by the rating agencies about its capability to face with financial commitments. The rating assignment of country $c$ at time $t$ is denoted by $x^{c}(t)$. The evaluation process carried out by the rating agencies depends on the financial, political and economic conditions experienced by the countries. Due to the uncertainty of these conditions, the rating variable can be considered as a realization of a stochastic process $X^{c}(t)=\left\{X^{c}(t), t \in \mathbb{R}^{+}\right\} \cdot X^{c}(t)$ takes values in the finite state space $E=\{1, \ldots, k\}$, with $k$ being the number of rating classes. At the same time, each country has to pay a given amount of credit spread on its financial obligations. The credit spread $r^{c}(t)$ is defined as the difference between the interest rate due by country $c$ at time $t$ and the minimum value of interest rates paid in $C$ at time $t$, i.e.:

$$
r^{c}(t):=i^{c}(t)-\min _{d \in C}\left\{i^{d}(t)\right\}
$$

In particular, the evolution of the credit spreads depends on the rating dynamics. Therefore, the spread is a realization of the stochastic process $R^{c}(t)=\left\{R^{c}(t), t \in \mathbb{R}^{+}\right\}$. To build the model, the following assumptions are advanced:

\section{Assumption 1}

The Stochastic Processes $X^{c}(t)$ are Independent and Identically Distributed (i.i.d.). We will Denote by $X(t)$ the Process Drawn from their Common Distributions.

Assumption 1 is justified by the need to build a simple and flexible model for estimation purposes. Moreover, due to the sparsity of data, the estimation of a correlation structure between countries may be a difficult task.

\section{Assumption 2}

The Stochastic Process $X(t)$ is a Continuous-Time Homogeneous Markov Chain.

It follows that $\forall s<t \in \mathbb{R}^{+}$:

$$
\begin{aligned}
& \mathbb{P}(X(t)=j \mid \sigma(X(h), h \leq s) X(s)=i) \\
& =\mathbb{P}(X(t)=j \mid X(s)=i)=p_{i j}(t-s) .
\end{aligned}
$$

Let us denote by $A=\left\{a_{i j}\right\}_{i, j \in E}$ the infinitesimal generator matrix whose elements $a_{i j} \geq 0$, for $i \neq j$ represent the transition intensities from state $i$ to state $j$ and $a_{i i}=-\sum_{j=1, i \neq j}^{k} a_{i j}$. The transition probability function $P(t)=\left\{p_{i j}(t)\right\}$ satisfies the Kolmogorov's Backward Equations:

$$
\frac{d}{d t} P(t)=A P(t)
$$

For the backward equations, we have the boundary initial condition $P(0)=I$. The solution of this initial value problem is given by:

$$
P(t)=e^{t A}:=\sum_{n=0}^{\infty} \frac{(t A)^{n}}{n !} .
$$

A complete treatment of Markov processes can be found in (Bremaud, 1981). Assumption 2 means that the future evolution of the process describing sovereign credit ratings is conditionally independent from the past, given the current state visited by the process (Markovian property) and that the transition intensities are constant over time. This assumption is supported by existent literature on credit risk models suggesting the use of Markov processes to describe rating dynamics (Trueck and Rachev, 2009). Furthermore, the continuous-time framework has a lot of advantages including the ability of capturing all transitions, including rare events; the capability to fit better with models that estimate yield curves (Christensen et al., 2004; Lando and Skødeberg, 2002; Jarrow et al., 1997). 


\section{Assumption 3}

The Process $R^{c}(t)$ Depends Exclusively on the Rating Dynamics. It does not Depend on the Previous Payment, nor on the time $t$, i.e. $\forall c, \forall x \in E, \forall t \in \mathbb{R}^{+}$:

$$
\begin{aligned}
& \mathbb{P}\left(R^{c}(t) \leq a \mid \sigma_{\infty}(X), \sigma_{t-}(R), X^{c}(t)=x\right) \\
& \mathbb{P}\left(R^{c}(t) \leq a \mid X^{c}(t)=x\right)
\end{aligned}
$$

where, $\sigma_{\infty}(X)$ is the sigma-algebra generated by the rating process over the time interval $[0,+\infty]$ and $\sigma_{t-}(R)$ is the sigma-algebra generated by the past payments of country $c$.

In assumption 3, the distribution of the credit spread paid by country $c$ at any given time $t$, conditional on the rating class occupied at the same time, is independent both on its previous payments and on the rating class occupied by other countries. This probability distribution is the same for all countries and times. As a consequence the probability distribution of the credit spread paid by each country $\left(R^{c}(t)\right)$ is a mixture of the possible allocation experienced by the country corresponding to the rating classes. More specifically:

$$
R^{c}(t) \stackrel{d}{=} \sum_{x=1}^{k} 1_{\left\{X^{c}(t)=x\right\}} R_{x},
$$

where, $1_{\{A\}}$ is the indicator function of the event $A$, while $\stackrel{d}{=} J$ means that the two random variables $I$ and $J$ have the same distribution. The random variables $R_{x}, x \in E$ are independently drawn from the distributions $F_{x}$ :

$$
F_{x}=\mathbb{P}\left(R_{x} \leq a\right)=\mathbb{P}\left(R^{c}(t) \leq a \mid X^{c}(t)=x\right) .
$$

Assumption 3 arises from the recognition about the influence of the rating dynamics on the credit spread evolution, which has been already pointed out in other works focusing on corporate bonds (Huang and Huang, 2012; D'Amico et al., 2011). This influence has been introduced in the model by the assumption of a common spread distribution for countries with the same rating assignment.

\section{Assumption 4}

There exist a vector of perturbations $S=\left(\lambda_{1}, \ldots \lambda_{k}\right)$ affecting the exit rates from each state of the Markov process, such that the matrix $A^{(\lambda)}=\left(a_{i j}^{(\lambda)}\right)$ given:

$$
\begin{aligned}
& a_{i j}^{(\lambda)}=a_{i j}+\lambda_{i}, i \neq j, i, j \in E, \\
& a_{i j}^{(\lambda)}=-\sum_{j=1, i \neq j}^{k} a_{i j}^{(\lambda)},
\end{aligned}
$$

\section{is a generator.}

The structured perturbations of Assumption 4 ensures the perturbed generator to be a transition rate matrix of a Markov process. The introduction of such perturbations is justified by the need to understand how the measure of inequality reacts to estimation errors and generally to some perturbations on the rating dynamics.

\section{The Dynamic Theil Entropy}

In this section we review shortly the dynamic Theil entropy as applied to assess the inequality of the financial risk distribution.

The inequality measure we used, has been based on the Theil index (Theil, 1967), which is computed as follows. Given the probability distribution built on the share of risk (credit spread) of each country $c$, i.e.:

$$
p(t)=\left\{p^{1}(t), \ldots, p^{N}(t)\right\},
$$

with:

$$
p^{c}(t)=\frac{r^{c}(t)}{\sum_{i=1}^{N} r^{i}(t)},
$$

the Theil index, in its deterministic formalization, is given by:

$$
\begin{aligned}
& T(p(t))=\log (N)-S(p(t)) \\
& =\sum_{i=1}^{N} p^{i}(t) \log \left(N p^{i}(t)\right):=\mathbb{K}(p(t) \mid u),
\end{aligned}
$$

where, $S(p(t))=-\sum_{i}^{N} p^{i}(t) \log p^{i}(t)$ is the Shannon entropy (for further information, Shannon, 1948). $T(p(t)$ ) coincides with the Kullback-Leibler divergence between the actual probability distribution $p(t)$ and the uniform one. It measures the degree of dispersion/concentration of the financial risk in the group of European countries, ranging between values equal to 0 and $\log (N)$. The first value is reached when there is a perfect equal distribution of payments among countries, i.e., $p^{c}(t)=$ $1 / N \forall \forall c \in \mathscr{C}$. The latter one is reached in the opposite situation when one country pays the total spread of the group, i.e., $p=\{0, \ldots, 1, \ldots 0\}$ for some $c$.

Under Assumptions 1-3 the inequality measure is a stochastic processes as it depends on the credit spreads which, in turns, are driven by the rating process. In this way, the inequality measure becomes a stochastic process too. A generalization for stochastic processes has been proposed in (D'Amico et al., 2012) and a decomposition has been successively advanced by (D'Amico et al., 2014). In the latter work, indeed, the authors derived a formula to assess the dynamic entropy by decomposing 
it into inter-group entropy and intra-group entropy. In the present work we apply the decomposed formula in a simplified scheme where the spreads paid by any country coincide with the average payment in each class. In this way, the only source of randomness is in the credit rating process that in turn generates credit spread payments. Therefore, we have to allocate the countries in $k$ groups, i.e., the rating classes $\{1, \ldots, k\}$.

Further, let denote by:

$$
n(t)=\left\{n_{1}(t), n_{2}(t), \ldots, n_{k}(t)\right\}
$$

the multivariate counting process, with $n_{i}(t)$ being the number of countries allocated in rating class $i$ and by:

$$
\operatorname{sh}(t)=\left\{s h_{1}(t), \ldots, s h_{k}(t)\right\},
$$

the probability distribution built on the share of credit spread paid by the rating classes, with:

$$
s h_{i}(t)=\frac{r_{i} \cdot n_{i}(t)}{\sum_{j=1}^{k} r_{j} \cdot n_{j}(t)},
$$

where, $r_{i}$ is the mean credit spread paid by class $i$. It should be noted that $r_{i}$ is supposed to be constant over time and for each rating class. The dynamic inequality is then computed according to Equation 3:

$$
\begin{aligned}
& \operatorname{DT}(p(t))=\sum_{i=1}^{k} s h_{i}(t) \cdot \operatorname{TE}\left(r_{i} ; n_{i}\right) \\
& +\sum_{i=1}^{k} s h_{i}(t) \cdot \log \left(k \cdot s h_{i}(t)\right) \\
& +\sum_{i=1}^{k} s h_{i}(t) \cdot \log \frac{N}{k \cdot n_{i}} .
\end{aligned}
$$

The first term consists of the intra-group inequality measure which results in a weighted average of the inequality computed within each rating class $i T E$ $\left(r_{i} ; n_{i}\right)$ and the share of risk of each class. In the present work, since all countries belonging to the same rating class $i$ pay the same credit spread $r_{i}$, the intra-group inequality is equal to zero. Accordingly, the dynamic inequality becomes:

$$
\begin{aligned}
& \operatorname{DT}(p(t))=\sum_{i=1}^{k} s h_{i}(t) \log \left(k \cdot s h_{i}(t)\right) \\
& +\sum_{i=1}^{k} s h_{i}(t) \cdot \log \frac{N}{k \cdot n_{i}} .
\end{aligned}
$$

The inter-group inequality is composed by the last two addenda of Equation (3). The second is the inequality measure assessed on the rating classes' share of risk and the last one summarizes the divergence of the actual distribution with respect to the case of a uniform allocation of countries in the $\mathrm{k}$ rating classes (for further details about the interpretation of this measure (D'Amico et al., 2014; 2018a)). The process is better summarized by means of its first order moment, which according to (D'Amico et al., 2014) is given by:

$$
\begin{aligned}
& \mathbb{E}[D T(p(t))] \\
& =\sum_{i=1}^{k} \sum_{\underline{n}^{\prime} \in p . c .} \frac{N !}{\prod_{h=1}^{k} n_{h}^{\prime} !} \prod_{h=1}^{k} P_{h}(t)^{n_{h}^{\prime}}[D T(p(t))],
\end{aligned}
$$

where:

$$
P_{h}(t)=\sum_{i=1}^{k} \frac{n_{i}(0)}{N} p_{i h}(t) .
$$

The symbol p.c. denotes the set of all possible configurations stemming from $\mathrm{N}$ countries and $\mathrm{k}$ rating classes. In particular:

$$
\text { | p.c. } \mid=\left(\begin{array}{c}
N+k-1 \\
k-1
\end{array}\right)
$$

\section{Simulations and Results}

In this section we evaluate how much the value of the dynamic Theil entropy changes responding to variations of the parameter $\lambda_{i}$. We performed a sensitivity analysis based on several simulations by varying the parameters and the way they affect the generator of the CTMC. To do so, we rely on structured perturbation (Lando and Skødeberg, 2002), by directly perturbing the parameters of the CTMC in such a way that a new Markov process is obtained having a generator matrix that is a perturbation of the initial generator. In this section we illustrate how the simulations are performed and the results.

\section{Simulation Scheme}

Given a vector of perturbations $S=\left(\lambda_{1}, \ldots \lambda_{k}\right)$ each perturbation is applied to the infinitesimal generator according to Assumption 4. In particular, once estimated the infinitesimal generator $A=\left\{a_{i j}\right\}_{i, j \in E}$ of the CTMC, we introduce the perturbation parameter to the generator. Then, we evaluate the difference between the dynamic inequality computed with the nominal model and that with the perturbed model. We denote by $\mathbb{E}\left[D T_{\lambda}(p(t))\right]$ the measure of inequality resulting by applying the vector of perturbations $S$ to the generator of the nominal model. The value of the perturbation $\lambda_{i}$ depends on the smallest value of the exit rate of each state in the CTMC. As a matter of fact: 


$$
\lambda_{i} \in\left(-\min \left(a_{i j}\right), \min \left(a_{i j}\right)\right)
$$

otherwise the resulting model will be no more a Markov chain and the perturbation will not be structured.

The simulations are carried out by firstly generating the entries of $S$ from a Normal distribution. Secondly, to include dependencies between rating classes, we generate perturbation values from a multivariate Normal distribution. This is done supposing three cases:

1. The perturbations affect all exit rates of the generator matrix, i.e., $a_{i j}^{(\lambda)}=a_{i j}+\lambda_{i}, \forall i \neq j$

2. The perturbations affect all upgrade intensities of the generator matrix, i.e., $a_{i j}^{(\lambda)}=a_{i j}+\lambda_{i}, \forall i>j$

3. The perturbations affect all downgrade intensities of the generator matrix, i.e., $a_{i j}^{(\lambda)}=a_{i j}+\lambda_{i}, \forall i<j$

In particular, the starting point for the simulations is the generator matrix of the CTMC. Therefore the generator matrix:

$$
A_{i j}=\left\{a_{i j}\right\}_{i, j \in E}
$$

is estimated from the observed rating data according to (Sadek and Limnios, 2005):

$$
\hat{a}_{i j}(t, N)=\left\{\begin{array}{cc}
\frac{n_{i j}}{v_{i}(t, N)} & i \neq j, v_{i}(t, N) \neq 0, \\
-\frac{\sum_{l \in E\{i\}} n_{i j}(t, N)}{v_{i}(t, N)} & i=j, v_{i}(t, N) \neq 0, \\
0 & v_{i}(t, N)=0,
\end{array}\right.
$$

where, $n_{i j}(t, N)$ is the number of total transitions from $i$ to $j$ observed for all countries over the time interval $[0, t]$ and $v_{i}(t, N)$ is the total time spent in rating class $i$ by all countries. Then, for each simulation we consider the perturbed generator and we compute $\mathbb{E}\left[D T_{\lambda}(p(t))\right]$. Given the huge amount of combinations required such for a computation ${ }^{1}$ we proceed with a Monte Carlo simulation both for the nominal and the perturbed model. For a number $R$ of Monte Carlo iterations the rating dynamics are generated according to a CTMC, given the generator

${ }^{1}$ The number of combinations stemming from 24 countries and 8 rating classes is:

$$
\text { p.c. }=\left(\begin{array}{c}
31 \\
7
\end{array}\right)=2629575
$$

$\hat{A}=\left\{\hat{a}_{i j}\right\}$ and the initial distribution. Therefore, the following sequence has to be built:

$$
T_{0}=0, X_{0}, T_{1}-T_{0}, X_{1}, \ldots, T_{n+1}-T_{n}, X_{n+1},
$$

where, $X_{n}$ denotes the rating class occupied at the $n$-th transition and $T_{n}-T_{n-1}$ represents the sojourn time between the (n-1)-th and $n$-th transition. The generation of a trajectory is done according to the following formulas:

$$
\left\{\begin{array}{l}
P\left(X_{n+1}=j \mid X_{0}=i_{0}, \ldots, X_{n}=i, T_{0}, \ldots, T_{n}\right) \\
=P\left(X_{n+1}=j \mid X_{n}=i\right)=\frac{a_{i j}}{a_{i}}, \\
P\left(T_{n+1}-T_{n} \leq x \mid X_{0}=i_{0}, \ldots, X_{n}=i, T_{0}, \ldots, T_{n}\right) \\
=P\left(T_{n+1}-T_{n} \leq x \mid X_{n}=i\right)=1-e^{-a_{i} x} .
\end{array}\right.
$$

Using current rating class, the next rating is simulated according to the probability distribution $\left(\frac{a_{i j}}{a_{i}}\right)$ while the sojourn time in rating $i$ according to an exponential distribution of parameter $a_{i}$. In particular, if $\left\{T_{n}\right\}$ is the increasing sequence of transition times of the process $X(t)$, then, by $X_{n}=X\left(T_{n}\right)$, we denote the embedded Markov chain:

$$
Q(i, j)=\mathbb{P}\left(X_{n+1}=j \mid X_{n}=i\right)= \begin{cases}\frac{a_{i j}}{a_{i}} & i \neq j, \\ 0 & i=j .\end{cases}
$$

which represents the probability of transition from state $i$ to state $j$ disregarding the sojourn time length. The latter follows an exponential distribution with parameters $a_{i}$. The evolution of payments is the simulated by assigning the mean of the empirical distributions of credit spread conditional on the rating class $\left(r_{i}\right)$. The dynamic Theil entropy is assessed as in Equation (3) on the simulated spreads and through the mean over all iterations we assess the dynamic entropy under the nominal model, i.e., $\mathbb{E}[D T(p(t))]$ and the dynamic entropy assessed with the perturbed generator, i.e., $\mathbb{E}\left[D T_{\lambda}(p(t))\right]$. In particular, for the perturbed model, a number of $P$ vectors of perturbations is generated and for each perturbation the Monte Carlo is performed. Computationally speaking, the resulting simulation algorithm is clearly embarrassingly parallel, that is almost always possible to obtain a linear speed up. The speed up is defined as:

$$
S=\frac{T_{1}}{T_{p}(n)}
$$


where, $T_{1}$ is the time needed to run the serial code and $T_{p}(n)$ is the time to run the same code in parallel using $n$ processes/treads/processors. In our case we always used a number of processes $n$ equal to the number of perturbations $P$.

\section{Results}

In this section we firstly give results of the dynamic entropy assessed under the nominal model, without perturbations and successively we presents the results of the simulations performed, each one introducing 100 different perturbations. As a starting point it is necessary to estimate the generator of the Markov process that describes the rating dynamic. The estimation is done according to estimator shown in Equation 5. The results are reported in Table 1 where the estimated generator is presented. As it is possible to see, the value of this matrix are little showing that rating changes occur unfrequently. The matrix is tridiagonal and reveals that changes in rating classes occur only from one class to the adjacent ones. Figure 3 shows the expected value of the dynamic Theil entropy computed with $R=100000$ iterations and for 365 days, whereas Table 2 gives results on the moments of the dynamic Theil entropy. The number of considered simulations is in agreement with (D'Amico et al., 2018a) where the authors have showed that it is an adequate number of iterations that guarantees the stability of the results.

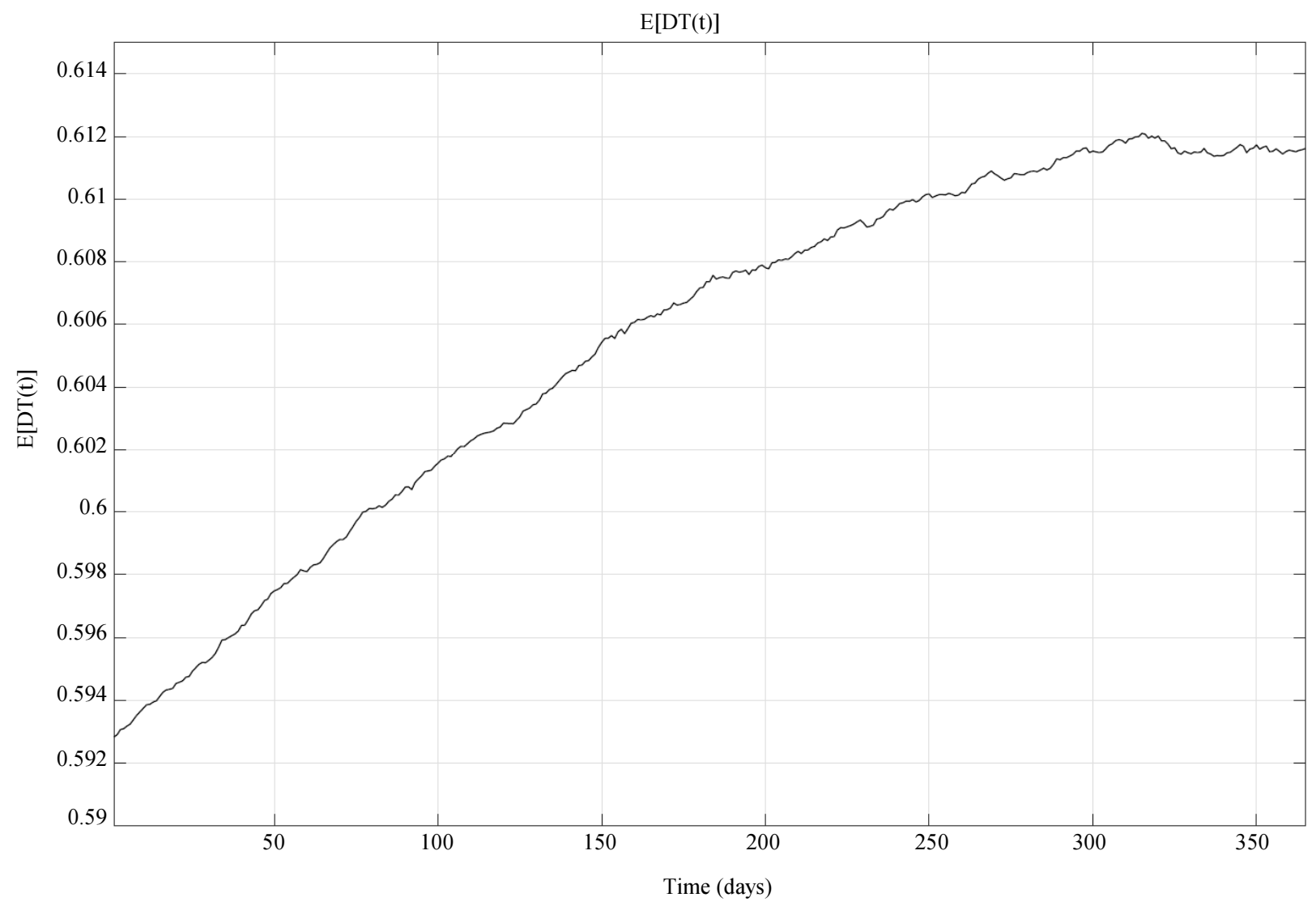

Fig. 3: Expected value of the dynamic entropy without any perturbations

Table 1: Estimate of the generator of the Markov process $\hat{A}=\left\{\hat{a}_{i j}\right\}_{i, j \in E}$

\begin{tabular}{lllllllll}
\hline & 1 & 2 & 3 & 4 & 5 & 6 & 7 & 8 \\
\hline 1 & $-1.897 \mathrm{e}-04$ & $1.897 \mathrm{e}-04$ & 0 & 0 & 0 & 0 & 0 & 0 \\
2 & $2.253 \mathrm{e}-04$ & $-4.13 \mathrm{e}-04$ & $1.877 \mathrm{e}-04$ & 0 & 0 & 0 & 0 & 0 \\
3 & 0 & $7.4 \mathrm{e}-05$ & $-4.069 \mathrm{e}-04$ & $3.329 \mathrm{e}-04$ & 0 & 0 & 0 & 0 \\
4 & 0 & 0 & $3.669 \mathrm{e}-04$ & $-6.115 \mathrm{e}-04$ & $2.446 \mathrm{e}-04$ & 0 & 0 & 0 \\
5 & 0 & 0 & 0 & $7.113 \mathrm{e}-04$ & $-8.129 \mathrm{e}-04$ & $1.016 \mathrm{e}-04$ & 0 & 0 \\
6 & 0 & 0 & 0 & 0 & $5.997 \mathrm{e}-04$ & $-1.1994 \mathrm{e}-03$ & $5.997 \mathrm{e}-04$ & 0 \\
7 & 0 & 0 & 0 & 0 & 0 & $1.5823 \mathrm{e}-03$ & $-4.7468 \mathrm{e}-03$ & $3.1646 \mathrm{e}-03$ \\
8 & 0 & 0 & 0 & 0 & 0 & $1.33 \mathrm{e}-02$ & $1.33 \mathrm{e}-02$ & $-2.67 \mathrm{e}-02$ \\
\hline
\end{tabular}


According to Fig. 3 and Table 2 the expected value of the Dynamic inequality shows an increasing trend, as well as its standard deviation, whereas the skewness and the kurtosis show high positive values which decrease over time. These empirical results suggest that, over the next year, the financial risk is more concentrated. This means, for instance, that some countries will pay more that the current time and this is caused by a deterioration of their creditworthiness.

To investigate how perturbations should impact on the assessment of the inequality measure, several simulations are performed supposing both the existence and the nonexistence of some contagion concerning the way the perturbations affect the rating classes' transition rates. To better evaluate the results, the higher order moments of the dynamic inequality resulting from the simulations are assessed. For the rest of the paper we will denote $\mathbb{E}$ $\left[D T_{\lambda}(p(t))\right]$ as $D T$ and $\mathbb{E}\left[D T_{\lambda}(p(t))\right]$ as $D T_{\lambda}$.

Case $\lambda_{i} \sim N(0,2.5 e-10)$

In this case the perturbation values are generated from a Normal distribution with $\mu=0, \sigma^{2}=2.5 \mathrm{e}-10$. The perturbations are then added to all off-diagonal entries and, alternatingly, only to the upgrades and only to the downgrade intensities. Table 3 and Fig. 4 give results of the simulations performed by adding the perturbation to all offdiagonal entries of $A=\left\{a_{i j}\right\}_{i, j \in E}$. For all Figures presented here, the solid black line denotes $D T$ whereas the dashed line represents the expected value of the dynamic entropy influenced by the perturbations, denoted as $\mu_{D T_{\lambda}}$.

$\mu_{D T_{\lambda}}$ is close the $D T$, the value of the standard deviation is increasing although is it very close to 0 . The skewness is positive for almost all times except in the period between $t=250$ and $t=300$ when it is negative. In fact, as it can be seen from Fig. 4, DT is not centralized and a large number of observations is concentrated on its right side. Finally, the values of the kurtosis are close to 3 (according to a Normal distribution).

When the perturbations affect only the downgrade intensities, the results are almost similar to the first simulation (as it can be seen from Fig. 5 in the first panel and Table 4), except for higher value of the standard deviation. On the other hand, when the perturbation is added only to the upgrade intensities, differences are encountered in the evolution of the skewness (Fig. 5, in the second panel and Table 5).

\section{Case $\lambda_{i} \sim N(0,5 e-9)$}

In this Section we present results of the simulations made supposing that the perturbation $\lambda_{i}$ follows a Normal distribution as in the previous section. The difference here is in the value of the variance which is larger and equal to 5e-9. Table 6 to 8 collect the descriptive statistics of $D T_{\lambda}$ when the perturbations are added to all exit rates, only to the downgrade and only to the upgrade rates, respectively.
Table 2: Descriptive statistics of $D T(p(t))$

\begin{tabular}{lllll}
\hline$t$ & 50 & 150 & 250 & 350 \\
\hline $\mathbb{E}[D T(p(t))]$ & 0.5975 & 0.6054 & 0.6102 & 0.6117 \\
$\sigma_{D T}(t)$ & 0.0410 & 0.0675 & 0.0806 & 0.0868 \\
skw & 5.3904 & 3.1780 & 2.5299 & 2.1954 \\
kurt & 35.19 & 13.472 & 9.2289 & 7.4557 \\
\hline
\end{tabular}

Table 3: Descriptive statistics of $D T_{\lambda}$ when $\lambda_{I} \sim N(0,2.5 \mathrm{e}-10)$

\begin{tabular}{lllll}
\multicolumn{5}{c}{ and $a_{i j}^{(\lambda)}=a_{i j}+\lambda_{i}, \forall a_{i j}>0}$. \\
\hline Day & 50 & 150 & 250 & 350 \\
\hline$\mu_{D T_{\lambda}}$ & 0.5981 & 0.6056 & 0.6096 & 0.6118 \\
$\sigma_{D T_{\lambda}}$ & 0.0015 & 0.0021 & 0.0029 & 0.0031 \\
skew & 1.0733 & 0.4864 & -0.3228 & 0.3861 \\
kurt & 2.7724 & 2.8192 & 3.1082 & 3.1667 \\
min & 0.5950 & 0.6008 & 0.6028 & 0.6052 \\
max & 0.6016 & 0.6105 & 0.6173 & 0.6221 \\
range & 0.0066 & 0.0097 & 0.0145 & 0.0170 \\
\hline
\end{tabular}

Table 4: Descriptive statistics of $D T_{\lambda}$ when $\lambda_{i} \sim N(0,2.5 \mathrm{e}-10)$ and $a_{i j}^{(\lambda)}=a_{i j}+\lambda_{i}, \forall i<j$

\begin{tabular}{lllll}
\hline Day & 50 & 150 & 250 & 350 \\
\hline$\mu_{D T_{\lambda}}$ & 0.5982 & 0.6058 & 0.6099 & 0.6115 \\
$\sigma_{D T_{\lambda}}$ & 0.0016 & 0.0027 & 0.0031 & 0.0032 \\
Skew & 1.0978 & 0.1591 & -0.2897 & 0.2169 \\
kurt & 2.8144 & 2.4289 & 2.5781 & 3.0752 \\
min & 0.5943 & 0.5996 & 0.6020 & 0.6049 \\
max & 0.6019 & 0.6111 & 0.6170 & 0.6215 \\
range & 0.0076 & 0.0116 & 0.0150 & 0.0166 \\
\hline
\end{tabular}

Table 5: Descriptive statistics of $D T_{\lambda}$ when $\lambda_{i} \sim N(0,2.5 \mathrm{e}-10)$

\begin{tabular}{lllll}
\multicolumn{5}{c}{ and $a_{i j}^{(\lambda)}=a_{i j}+\lambda_{i}, \forall i>j$} \\
\hline Day & 50 & 150 & 250 & 350 \\
\hline$\mu_{D T_{\lambda}}$ & 0.5978 & 0.6053 & 0.6090 & 0.6112 \\
$\sigma_{D T_{\lambda}}$ & 0.0014 & 0.0022 & 0.0031 & 0.0033 \\
skew & 1.0986 & -0.4012 & -0.7451 & -0.4063 \\
kurt & 3.6708 & 3.1403 & 2.1745 & 2.4632 \\
min & 0.5949 & 0.5988 & 0.6028 & 0.6040 \\
max & 0.6020 & 0.6109 & 0.6154 & 0.6194 \\
range & 0.0073 & 0.0121 & 0.0126 & 0.0154 \\
\hline
\end{tabular}

Table 6: Descriptive statistics of $D T_{\lambda}$ when $\lambda_{i} \sim N(0,5 \mathrm{e}-09)$ and $a_{i j}^{(\lambda)}=a_{i j}+\lambda_{i}, \forall i \neq j$

\begin{tabular}{lllll}
\hline Day & 50 & 150 & 250 & 350 \\
\hline$\mu_{D T_{\lambda}}$ & 0.5980 & 0.6051 & 0.6091 & 0.6109 \\
$\sigma_{D T_{\lambda}}$ & 0.0014 & 0.0026 & 0.0034 & 0.0039 \\
skew & 0.9913 & -0.4474 & -0.8191 & -0.6173 \\
kurt & 3.1146 & 2.7112 & 2.2092 & 2.4256 \\
min & 0.5944 & 0.5983 & 0.6027 & 0.6024 \\
max & 0.6018 & 0.6110 & 0.6158 & 0.6198 \\
range & 0.0074 & 0.0127 & 0.0131 & 0.0174 \\
\hline
\end{tabular}


Table 7: Descriptive statistics of $D T_{\lambda}$ when $\lambda_{i} \sim N(0 ; 5 \mathrm{e}-09)$ and $a_{i j}^{(\lambda)}=a_{i j}+\lambda_{i}, \forall i<j$.

\begin{tabular}{lllll}
\hline Day & 50 & 150 & 250 & 350 \\
\hline$\mu_{D T_{\lambda}}$ & 0.5980 & 0.6054 & 0.6098 & 0.6116 \\
$\sigma_{D T_{\lambda}}$ & 0.0017 & 0.0033 & 0.0047 & 0.0059 \\
skew & 1.0334 & 0.1434 & -0.1462 & -0.1211 \\
kurt & 3.0501 & 2.3170 & 2.4323 & 2.3164 \\
min & 0.5949 & 0.5975 & 0.6008 & 0.5981 \\
max & 0.6027 & 0.6125 & 0.6223 & 0.6250 \\
range & 0.0078 & 0.0150 & 0.0215 & 0.0269 \\
\hline
\end{tabular}

Table 8: Descriptive statistics of $D T_{\lambda}$ when $\lambda_{i} \sim N(0,5 \mathrm{e}-09)$ and $a_{i j}^{(\lambda)}=a_{i j}+\lambda_{i}, \forall i>j$.

\begin{tabular}{lllll}
\hline Day & 50 & 150 & 250 & 350 \\
\hline$\mu_{D T_{\lambda}}$ & 0.5978 & 0.6049 & 0.6088 & 0.6104 \\
$\sigma_{D T_{\lambda}}$ & 0.0015 & 0.0028 & 0.0041 & 0.0050 \\
skew & 0.8862 & -0.2280 & -0.7333 & -0.5258 \\
kurt & 3.2791 & 2.3371 & 2.5009 & 2.5985 \\
min & 0.5946 & 0.5985 & 0.5990 & 0.5991 \\
max & 0.6025 & 0.6108 & 0.6177 & 0.6227 \\
range & 0.0078 & 0.01245 & 0.0187 & 0.0236 \\
\hline
\end{tabular}

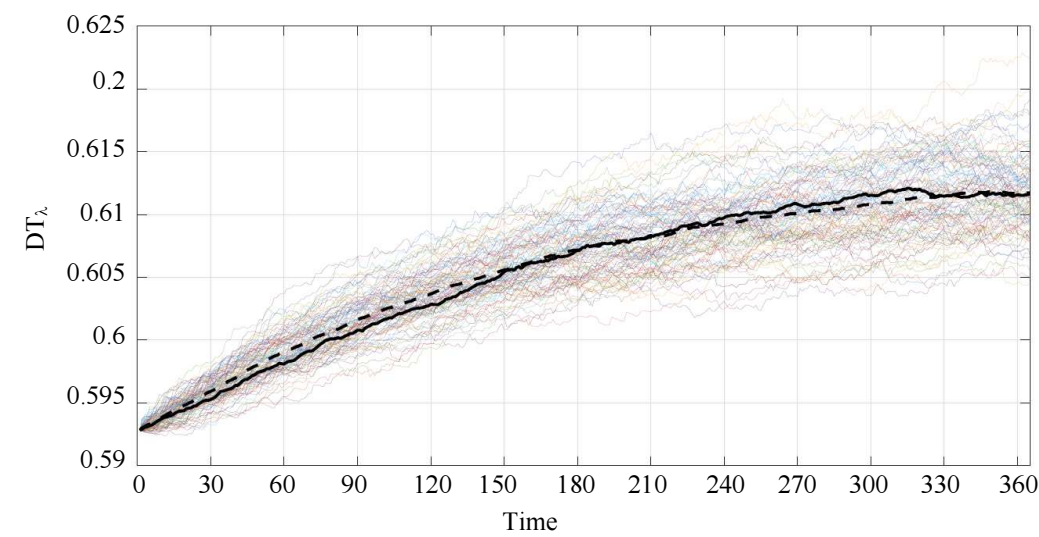

Fig. 4: Simulations when $\lambda_{i} \sim N(0,2.5 \mathrm{e}-10)$
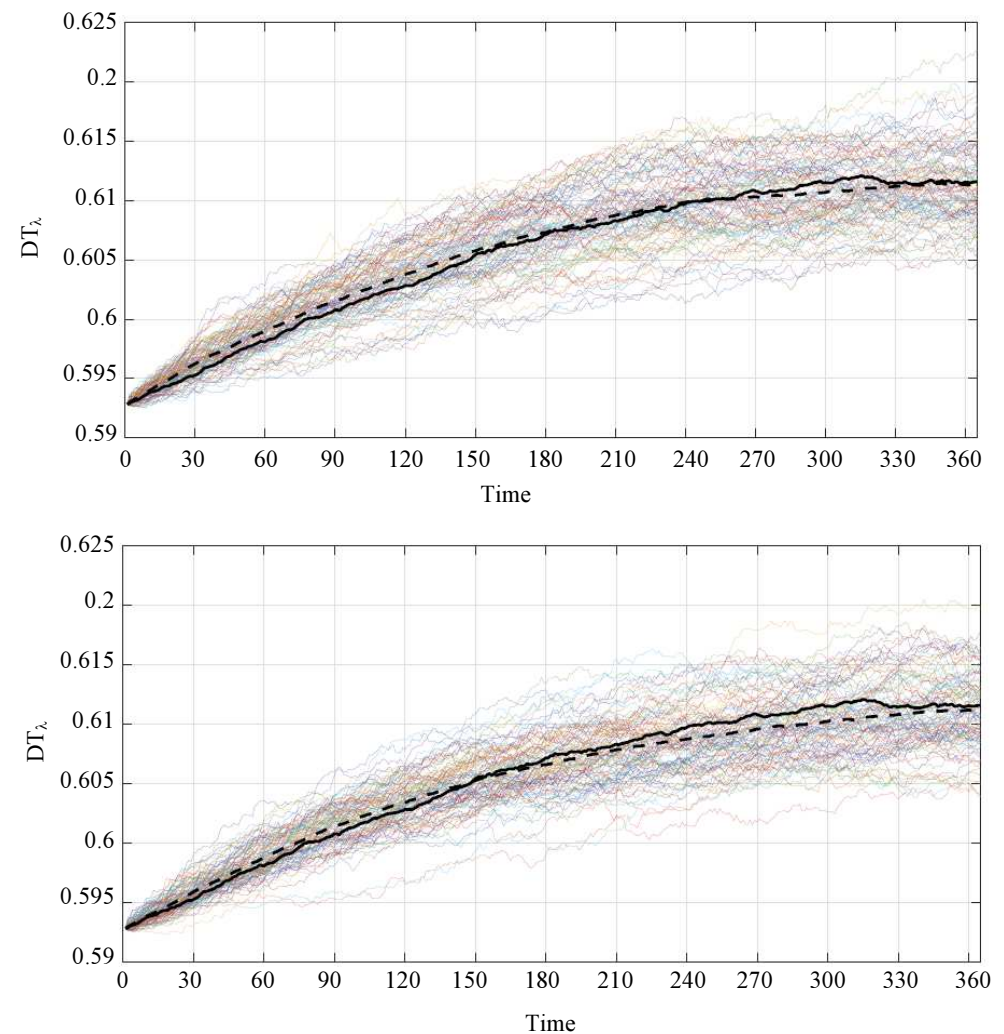

Fig. 5: Simulations when $\lambda \sim N(0,2.5 \mathrm{e}-10)$ and it is applied to downgrade/upgrade rates in the first/second panel 


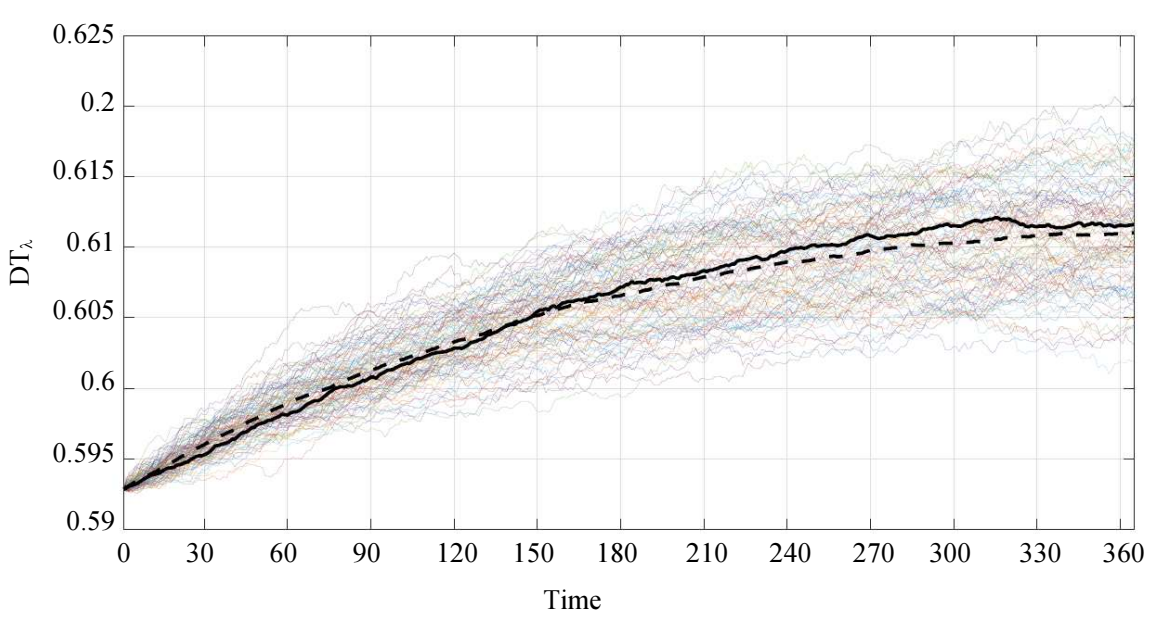

Fig. 6: $a_{i j}^{(\lambda)}=a_{i j}+\lambda_{i}, \forall i \neq j$

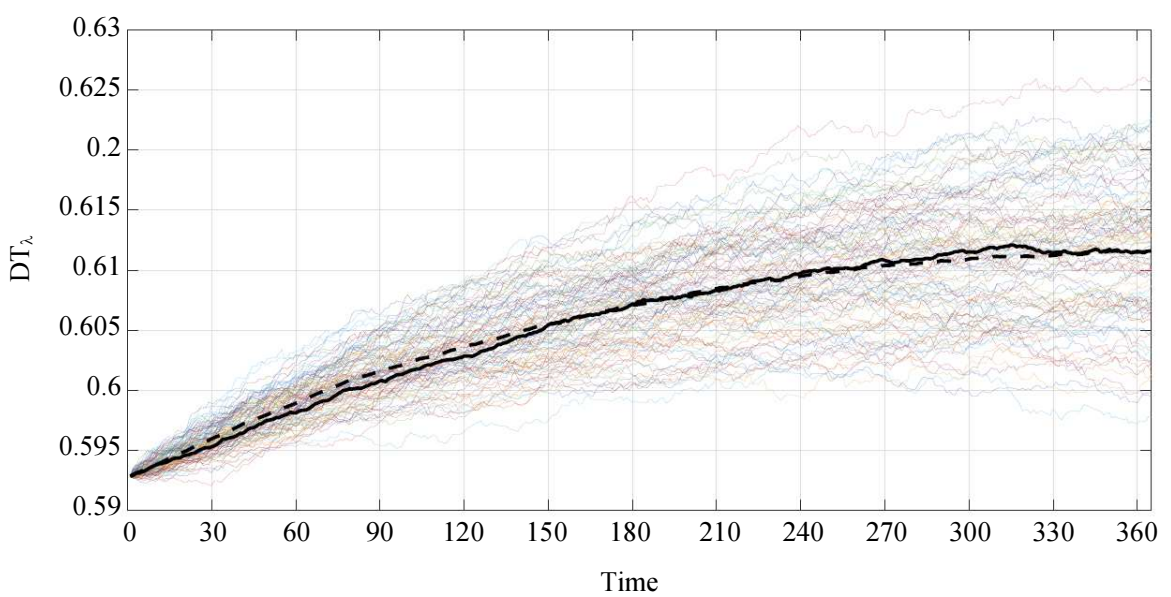

Fig. 7: $a_{i j}^{(\lambda)}=a_{i j}+\lambda_{i}, \forall i<j$

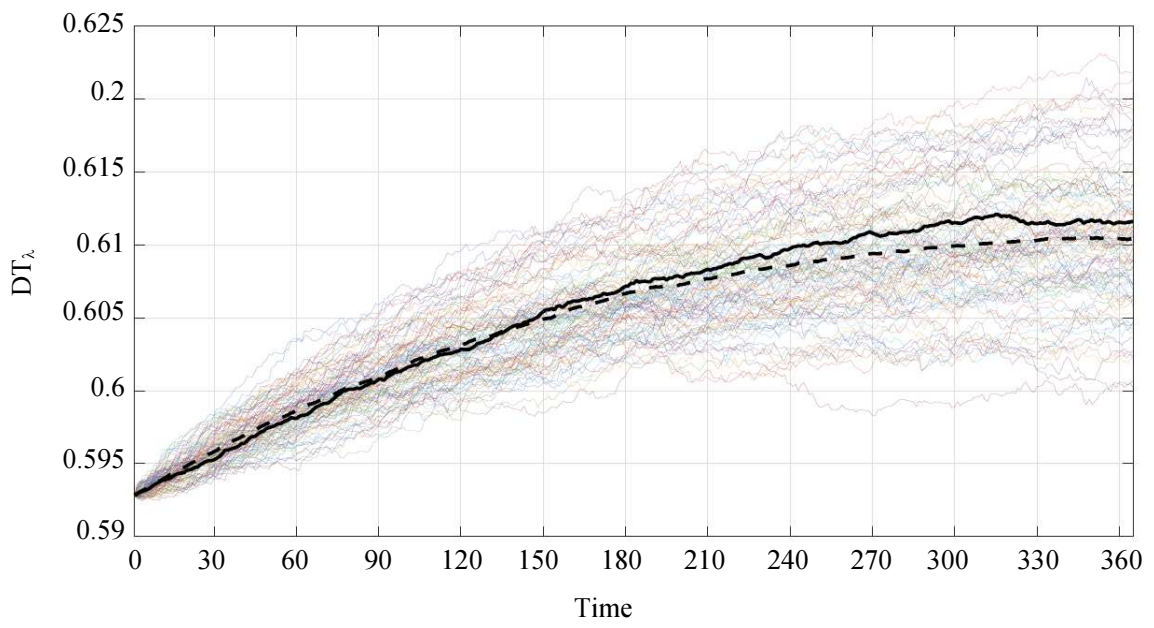

Fig. 8: $a_{i j}^{(\lambda)}=a_{i j}+\lambda_{i}, \forall i>j$ 
Whereas Fig. 6 to 8 show the plot of the simulations in terms of $D T_{\lambda}$ for all 100 perturbations (the expected value is represented by a dashed black line), compared with $D T$ (solid black line) According to the results we observe that by giving a larger variance, the results are similar to the first situation except for a higher dispersion around the expected value, confirmed by the larger values of the standard deviation of $D T_{\lambda}$ in all three cases. We also observe that when the variance of the perturbation is higher then the range of values of $D T_{\lambda}$ tends to increase with an amplifications of possible values of the financial inequality.

\section{Case $\lambda_{i} \sim N(0, \Sigma)$}

In this subsection we introduce a dependence among the rating classes while performing the simulations for the perturbed model. More specifically, the perturbation parameter $\lambda_{i}$ are generated according to a multivariate Normal distribution with zero mean and the covariance matrix shown in Table 9. The variance is the same as that proposed in the previous case. However, we introduce different covariance values for investment and speculative rating classes where the covariance between investment and speculative rating classes represents the smallest value. Figures 9 to 11 show the plots of the simulations in terms of $D T_{\lambda}$ for all 100 perturbations in all three cases. While Table 10 to 12 give results about the descriptive statistics of $D T_{\lambda}$ for all three cases. When the perturbations are added to all exit rates of the generator matrix, $D T_{\lambda}$ is closer to $D T$ with respect to the case without dependence among rating classes. Furthermore, the value of the skewness, which in the previous simulations was decreasing, shows now an alternating trend. Finally, the kurtosis are close to 3 (according to a Normal distribution) as well as the case with $\lambda_{i} \sim N(0,2.5 \mathrm{e}-$ $10)$, but different from the case with $\lambda_{i} \sim N(0,5 \mathrm{e}-9)$. When the perturbations affect only the downgrade rates of $A=\left\{a_{i j}\right\}_{i, j \in E}$, differences are encountered in the skewness and kurtosis values. They, in fact, show an alternating trends, which are dissimilar from what we have found in the simulation with $\lambda_{i} \sim N(0,5 \mathrm{e}-9)$. Finally, when the perturbations influences only the upgrade rates of $A=\left\{a_{i j}\right\}_{i, j \in E}$ the values of $\sigma_{D T_{2}}$ are smaller than when the perturbations affect the downgrade intensities even if the differences are very small. Regarding the skewness, it shows positive value up to $t=300$, which is different from the case without dependence, when the skewness becomes negative starting from $t=150$. But the main difference concerns the kurtosis, whose value are very high with respect to all other cases.
Case $\lambda_{i} \sim N(0, \Sigma)$, with $\Sigma_{i, j}<0, \quad i \in\{1,2,3,4\}$, $j \in\{5,6,7,8\}$

The latest simulations are performed by including a negative covariance between investment grade rating classes and speculative grade rating classes. In particular, we imposed $\Sigma_{i, j}=-1 e-10$, with $i$ and $j$ denoting, respectively, the investment rating classes and the speculative rating classes. The resulting covariance matrix is showed in Table 13. Figure 12 to 14 show the results of the simulations while Table 14 to 16 give results about the descriptive statistics of $D T_{\lambda}$ for all three cases. When the perturbations are added to all exit rates of the generator matrix, the difference between $\mu_{D T_{\lambda}}$ and $D T$ is higher with respect to the simulations illustrated in the previous subsection, so as the standard deviation. Regarding the skewness, as it can be seen in Table 10 and 14, it shows a similar trend with respect to the previous simulations, even if it becomes negative before $(t=$ 150). In the second case, the differences between $\mu_{D T_{\lambda}}$ and DT, along with $\sigma_{D T_{\lambda}}$, are more pronounced with respect to the other two cases and to the simulations exposed in the previous subsection. Finally, the third case shows the lowest values of $\sigma_{D T_{2}}$, but there are no crucial differences compared with the corresponding case of the previous subsection. The kurtosis is also different: It shows a different trend and lower values. In this case it is, in fact, firstly decreasing and successively increasing whereas with all element of the covariance matrix being positive, it shows an opposite trend.

To sum up, results of the simulations highlight the fact that the dynamic Theil index reacts differently and dependently on the type of the perturbations. As a matter of example:

- $\quad \mu_{D T_{\lambda}}$ is closer to DT when $a_{i j}^{(\lambda)}=a_{i j}+\lambda_{i}, \forall i<j$ with respect to the two other cases

- When $a_{i j}^{(\lambda)}=a_{i j}+\lambda_{i}, \forall i>j$, the skewness in negative for most of the simulated time whereas in the two other cases it becomes negative almost at the end

- By including the dependence among rating classes, the dissimilarities on the skewness described in the previous point are mitigated

- $\mu_{D T_{\lambda}}$ is closer to $D T$ when the perturbation is generated from a multivariate normal distribution than in the other cases

- There are also differences in the kurtosis while looking at the simulation with dependence among rating classes, most of all considering the variations of upgrade intensities 


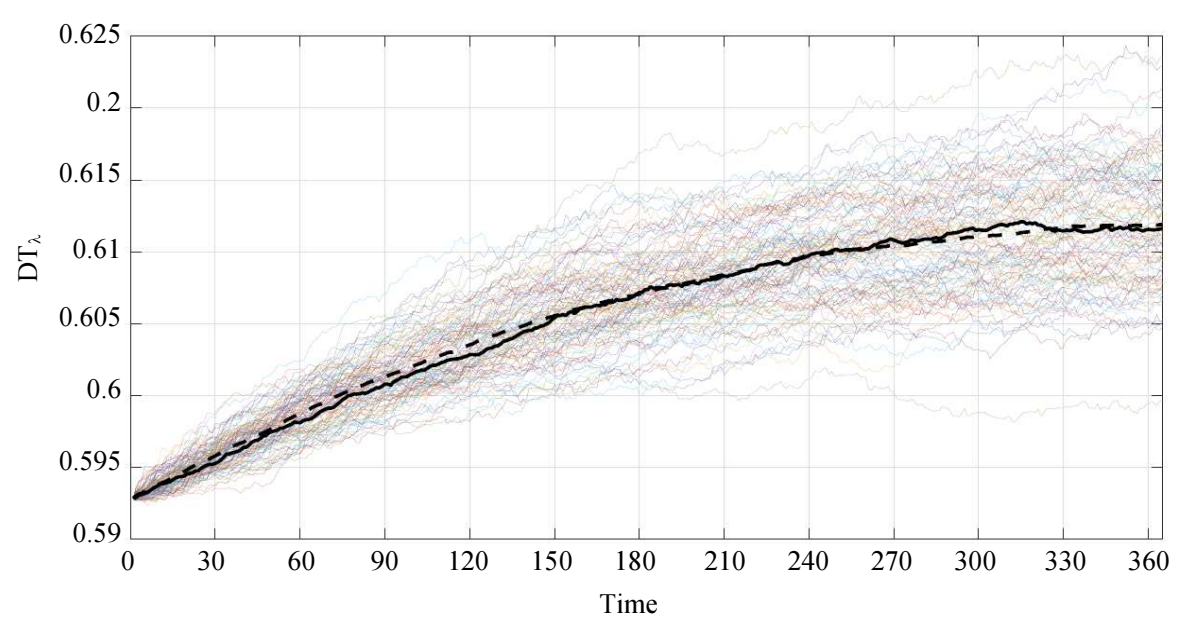

Fig. 9: $a_{i j}^{(\lambda)}=a_{i j}+\lambda_{i}, \forall i \neq j$

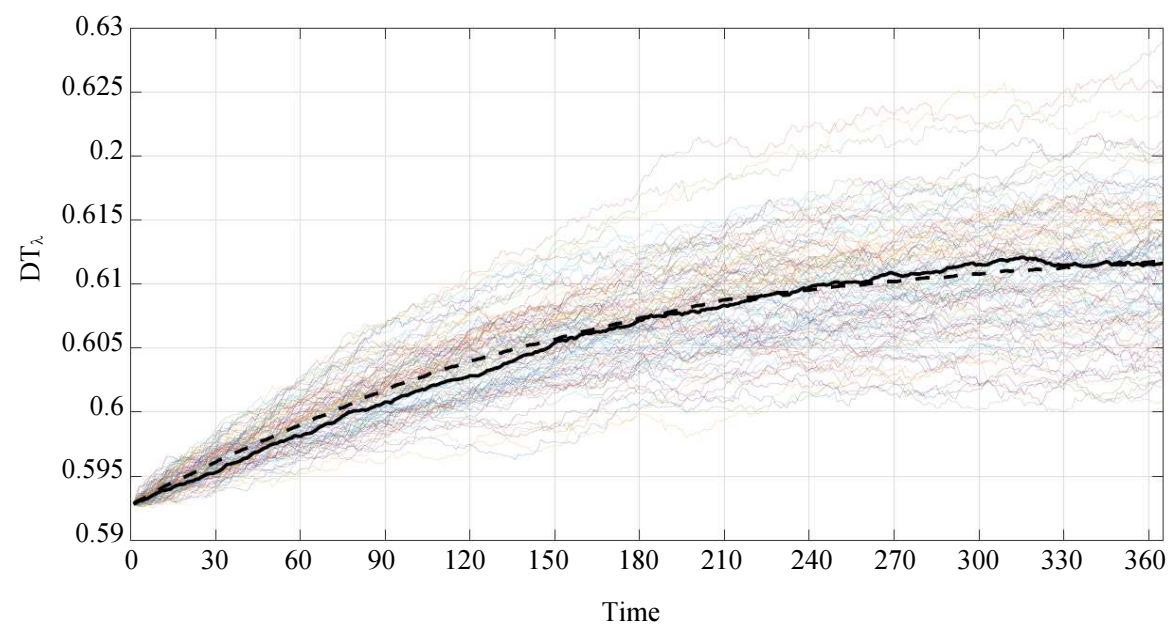

Fig. 10: $a_{i j}^{(\lambda)}=a_{i j}+\lambda_{i}, \forall i<j$

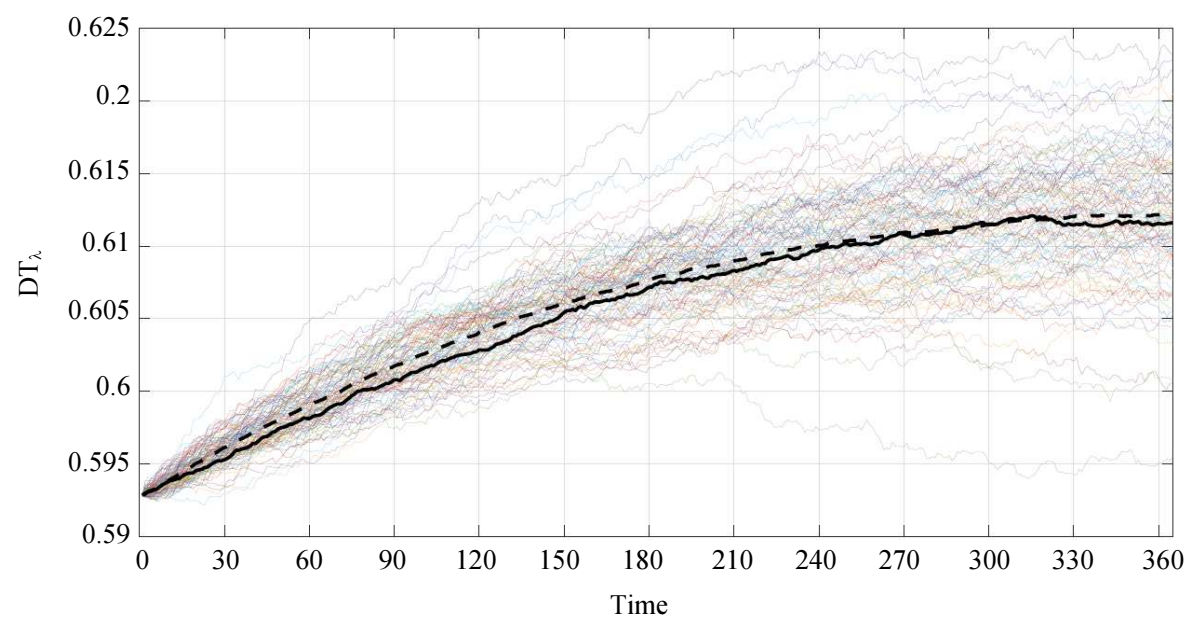

Fig. 11: $a_{i j}^{(\lambda)}=a_{i j}+\lambda_{i}, \forall i>j$ 


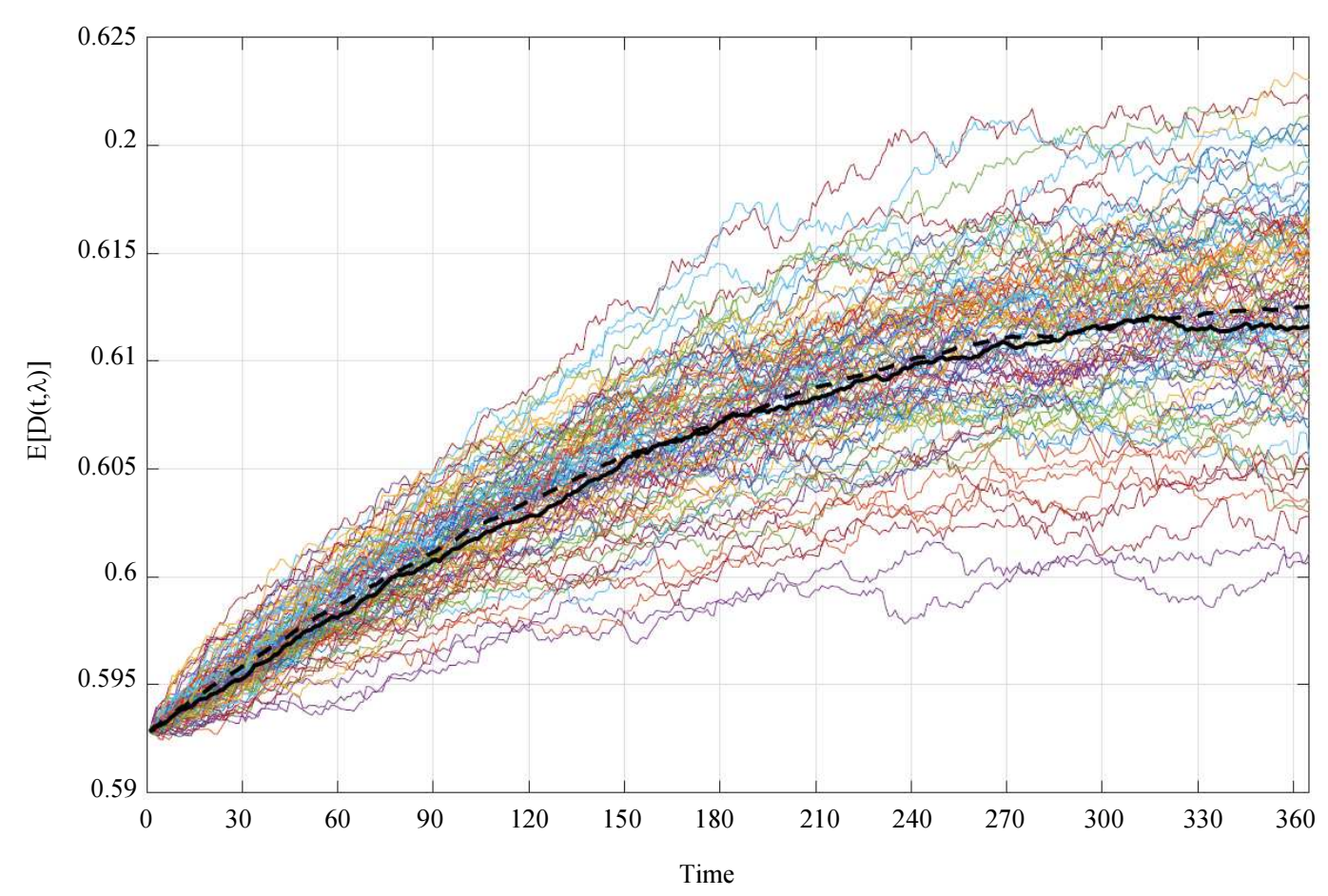

Fig. 12: $a_{i j}^{(\lambda)}=a_{i j}+\lambda_{i}, \forall i \neq j$

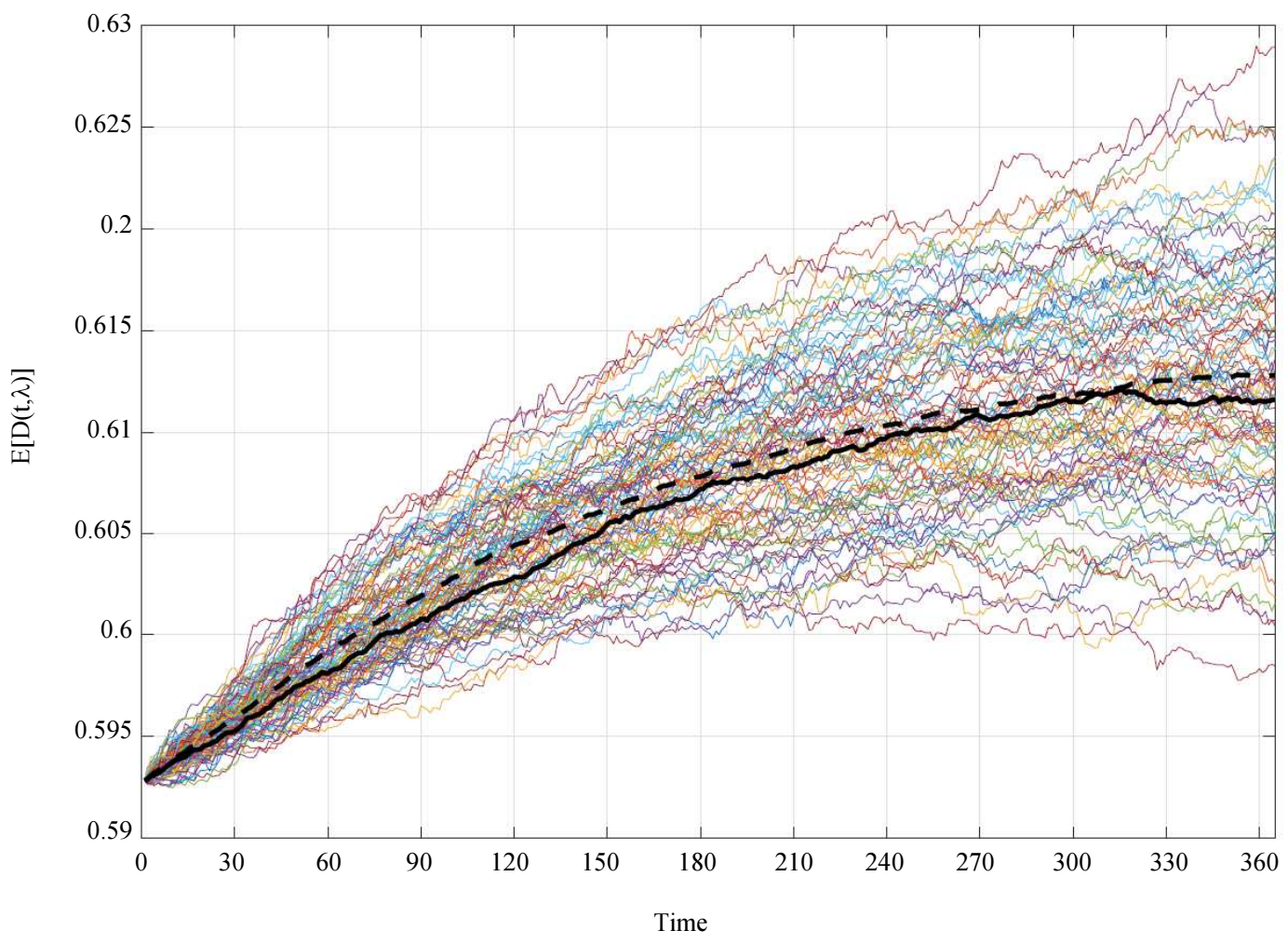

Fig. 13: $a_{i j}^{(\lambda)}=a_{i j}+\lambda_{i}, \forall i<j$ 


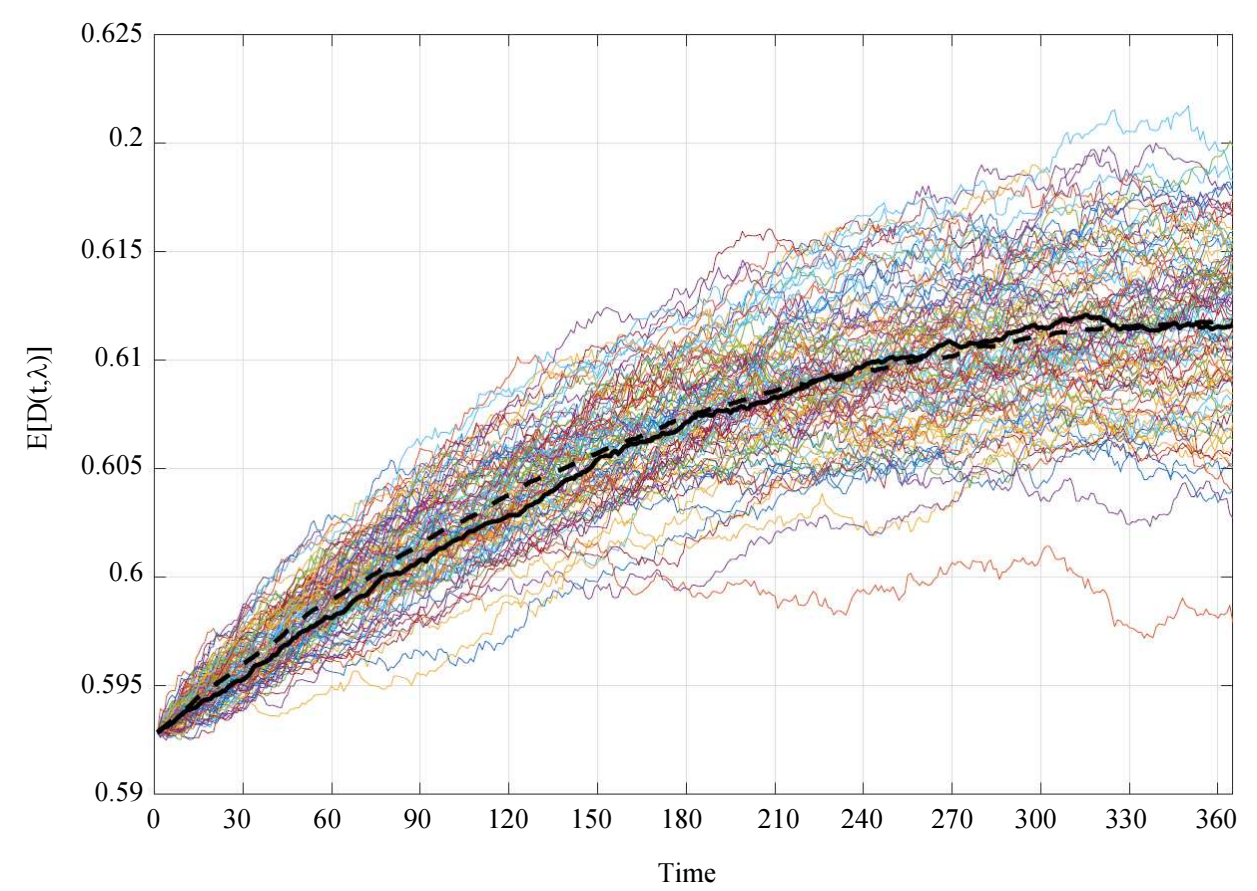

Fig. 14: $a_{i j}^{(\lambda)}=a_{i j}+\lambda_{i}, \forall i>j$

Table 9: Covariance matrix

\begin{tabular}{|c|c|c|c|c|c|c|c|c|}
\hline $\mathrm{k}$ & 1 & 2 & 3 & 4 & 5 & 6 & 7 & 8 \\
\hline 1 & $5 e-9$ & $2.5 \mathrm{e}-10$ & $2.5 \mathrm{e}-10$ & $2.5 \mathrm{e}-10$ & $1 \mathrm{e}-10$ & $1 \mathrm{e}-10$ & $1 \mathrm{e}-10$ & 1e-10 \\
\hline 2 & & $5 e-9$ & $2.5 \mathrm{e}-10$ & $2.5 \mathrm{e}-10$ & $1 \mathrm{e}-10$ & $1 \mathrm{e}-10$ & $1 \mathrm{e}-10$ & $1 e-10$ \\
\hline 3 & & & $5 e-9$ & $2.5 \mathrm{e}-10$ & $1 \mathrm{e}-10$ & $1 \mathrm{e}-10$ & $1 \mathrm{e}-10$ & $1 e-10$ \\
\hline 4 & & & & $5 e-9$ & $1 \mathrm{e}-10$ & $1 \mathrm{e}-10$ & $1 \mathrm{e}-10$ & $1 e-10$ \\
\hline 5 & & & & & $5 e-9$ & $4 \mathrm{e}-10$ & $4 \mathrm{e}-10$ & $4 e-10$ \\
\hline 6 & & & & & & $5 e-9$ & $4 \mathrm{e}-10$ & $4 \mathrm{e}-10$ \\
\hline 7 & & & & & & & $5 e-9$ & $4 \mathrm{e}-10$ \\
\hline 8 & & & & & & & & $5 e-9$ \\
\hline
\end{tabular}

Table 10: Descriptive statistics of $D T_{\lambda}$ when $\lambda_{i} \sim N(0, \Sigma)$ and $a_{i j}^{(\lambda)}=a_{i j}+\lambda_{i}, \forall i \neq j$

\begin{tabular}{lllll}
\hline Day & 50 & 150 & 250 & 350 \\
\hline$\mu_{D T_{\lambda}}$ & 0.5977 & 0.6055 & 0.6100 & 0.6119 \\
$\sigma_{D T_{\lambda}}$ & 0.0013 & 0.0029 & 0.0035 & 0.0044 \\
skew & 0.4848 & 0.2970 & -0.1620 & 0.2435 \\
kurt & 3.3276 & 2.5097 & 2.5800 & 2.9974 \\
min & 0.5940 & 0.6000 & 0.6013 & 0.5992 \\
max & 0.6015 & 0.6124 & 0.6188 & 0.6236 \\
range & 0.0075 & 0.0124 & 0.0175 & 0.0244 \\
\hline
\end{tabular}

Table 11: Descriptive statistics of $D T_{\lambda}$ when $\lambda_{i} \sim N(0, \Sigma)$ and $a_{i j}^{(\lambda)}=a_{i j}+\lambda_{i}, \forall i<j$

\begin{tabular}{lllll}
\hline Day & 50 & 150 & 250 & 350 \\
\hline$\mu_{D T_{\lambda}}$ & 0.5981 & 0.6057 & 0.6098 & 0.6115 \\
$\sigma_{D T_{\lambda}}$ & 0.0016 & 0.0034 & 0.0045 & 0.0054 \\
skew & 0.9971 & 0.1355 & 0.1044 & 0.0905 \\
kurt & 2.7638 & 2.9221 & 2.8739 & 2.7938 \\
min & 0.5942 & 0.5974 & 0.6008 & 0.6009 \\
max & 0.6020 & 0.6138 & 0.6227 & 0.6265 \\
range & 0.0078 & 0.0164 & 0.0219 & 0.0256 \\
\hline
\end{tabular}


Guglielmo D'Amico et al. / Journal of Mathematics and Statistics 2019, Volume 15: 280.297 DOI: $10.3844 / j m s s p .2019 .280 .297$

Table 12: Descriptive statistics of $D T_{\lambda}$ when $\lambda_{i} \sim N(0, \Sigma)$ and $a_{i j}^{(\lambda)}=a_{i j}+\lambda_{i}, \forall i>j$

\begin{tabular}{lllll}
\hline Day & 50 & 150 & 250 & 350 \\
\hline$\mu_{D T_{\lambda}}$ & 0.5981 & 0.6060 & 0.6104 & 0.6121 \\
$\sigma_{D T_{\lambda}}$ & 0.0015 & 0.0027 & 0.0039 & 0.0046 \\
skew & 1.0644 & 1.0703 & 0.3811 & -0.2450 \\
kurt & 3.2191 & 3.9959 & 4.9079 & 3.9667 \\
min & 0.5943 & 0.6011 & 0.5972 & 0.5960 \\
max & 0.6024 & 0.6149 & 0.6231 & 0.6238 \\
range & 0.0081 & 0.0138 & 0.0259 & 0.0278 \\
\hline
\end{tabular}

Table 13: Covariance matrix

\begin{tabular}{lllllllll}
\hline $\mathrm{k}$ & 1 & 2 & 3 & 4 & 5 & 6 & 7 & 8 \\
\hline 1 & $5 \mathrm{e}-9$ & $2.5 \mathrm{e}-10$ & $2.5 \mathrm{e}-10$ & $2.5 \mathrm{e}-10$ & $-1 \mathrm{e}-10$ & $-1 \mathrm{e}-10$ & $-1 \mathrm{e}-10$ & $-1 \mathrm{e}-10$ \\
2 & & $5 \mathrm{e}-9$ & $2.5 \mathrm{e}-10$ & $2.5 \mathrm{e}-10$ & $-1 \mathrm{e}-10$ & $-1 \mathrm{e}-10$ & $-1 \mathrm{e}-10$ & $-1 \mathrm{e}-10$ \\
3 & & $5 \mathrm{e}-9$ & $2.5 \mathrm{e}-10$ & $-1 \mathrm{e}-10$ & $-1 \mathrm{e}-10$ & $-1 \mathrm{e}-10$ & $-1 \mathrm{e}-10$ \\
4 & & & $5 \mathrm{e}-9$ & $-1 \mathrm{e}-10$ & $-1 \mathrm{e}-10$ & $-1 \mathrm{e}-10$ & $-1 \mathrm{e}-10$ \\
5 & & & & $5 \mathrm{e}-9$ & $4 \mathrm{e}-10$ & $4 \mathrm{e}-10$ & $4 \mathrm{e}-10$ \\
6 & & & & & $5 \mathrm{e}-9$ & $4 \mathrm{e}-10$ & $4 \mathrm{e}-10$ \\
7 & & & & & & & $5 \mathrm{e}-9$ & $4 \mathrm{e}-10$ \\
8 & & & & & & & $5 \mathrm{e}-9$ \\
\hline
\end{tabular}

Table 14: Descriptive statistics of $D T_{\lambda}$ when $\lambda_{i} \sim N(0, \Sigma)$ and $a_{i j}^{(\lambda)}=a_{i j}+\lambda_{i}, \forall i \neq j$

\begin{tabular}{lllll}
\hline Day & 50 & 150 & 250 & 350 \\
\hline$\mu_{D T_{\lambda}}$ & 0.5978 & 0.6056 & 0.6104 & 0.6124 \\
$\sigma_{D T_{\lambda}}$ & 0.0015 & 0.0030 & 0.0037 & 0.0046 \\
skew & 0.5379 & -0.2176 & -0.2580 & 0.1311 \\
kurt & 3.0695 & 2.8560 & 3.4653 & 2.8239 \\
min & 0.5937 & 0.5977 & 0.5986 & 0.5999 \\
max & 0.6014 & 0.6127 & 0.6192 & 0.6225 \\
range & 0.0077 & 0.0150 & 0.0205 & 0.0226 \\
\hline
\end{tabular}

Table 15: Descriptive statistics of $D T_{\lambda}$ when $\lambda_{i} \sim N(0, \Sigma)$ and $a_{i j}^{(\lambda)}=a_{i j}+\lambda_{i}, \forall i<j$

\begin{tabular}{lllll}
\hline Day & 50 & 150 & 250 & 350 \\
\hline$\mu_{D T_{\lambda}}$ & 0.5981 & 0.6062 & 0.6106 & 0.6127 \\
$\sigma_{D T_{\lambda}}$ & 0.0017 & 0.0033 & 0.0047 & 0.0059 \\
skew & 1.1635 & 0.8669 & 0.3325 & 0.5140 \\
kurt & 2.6469 & 2.7366 & 2.2493 & 2.6975 \\
min & 0.5951 & 0.6000 & 0.6002 & 0.5984 \\
max & 0.6015 & 0.6134 & 0.6201 & 0.6271 \\
range & 0.0064 & 0.0135 & 0.0200 & 0.0286 \\
\hline
\end{tabular}

Table 16: Descriptive statistics of $D T_{\lambda}$ when $\lambda_{i} \sim N(0, \Sigma)$ and $a_{i j}^{(\lambda)}=a_{i j}+\lambda_{i}, \forall i>j$

\begin{tabular}{lllll}
\hline Day & 50 & 150 & 250 & 350 \\
\hline$\mu_{D T_{\lambda}}$ & 0.5981 & 0.6057 & 0.6098 & 0.6118 \\
$\sigma_{D T_{\lambda}}$ & 0.0015 & 0.0026 & 0.0034 & 0.0040 \\
skew & 1.0960 & 0.2698 & -0.3920 & -0.2167 \\
kurt & 3.0254 & 2.7170 & 2.9179 & 3.0725 \\
min & 0.5942 & 0.5996 & 0.5995 & 0.5986 \\
max & 0.6015 & 0.6123 & 0.6167 & 0.6217 \\
range & 0.0073 & 0.0126 & 0.0172 & 0.0231 \\
\hline
\end{tabular}




\section{Concluding Remarks}

In this work we investigate the sensitivity of the dynamic Theil index computed on the credit spread process with respect to perturbations affecting the underlying rating dynamics. Starting from the model proposed in (D'Amico et al., 2018b), we consider different structured perturbations to the generator and we perform several simulations to evaluate how the inequality measure changes to different perturbations. The methodology is applied to real data concerning long-term interest rate on government bonds and sovereign credit ratings assigned by $\mathrm{S} \& \mathrm{P}$, whereas the perturbations are arbitrarily chosen. Obtained results suggest different sensitivity of the inequality measure to perturbations in the different presented scenarios, as there are difference when the perturbations affect all off-diagonal rates, only the upgrade rates and only the downgrade rates, respectively. The dependencies among ratings classes play a crucial role too, that should be more extensively investigated. To conclude, this study could be used to understand the impact on a financial system of the occurrence of some perturbations represented by estimation errors in credit risk models, with a particular focus on the inequality measure. Possible extension include a more sophisticated model that could take into account all the possible scenarios we presented in the simulations and also the consideration of higher order correlation in the perturbation scheme.

\section{Acknowledgment}

The authors gratefully acknowledge the constructive comments and suggestions of the reviewers which resulted in much improved paper.

\section{Author's Contributions}

Guglielmo D'Amico: Supervised the research. He was responsible of the conception, design and organization of the study. He reviewed the manuscript.

Stefania Scocchera: Contributed to the writing of the manuscript, analyzed data and constructed the software. She partecipated to all the experiments.

Loriano Storchi: Analyzed data and constructed the software. He partecipated to all the experiments.

\section{Ethics}

The authors declare that there is no conflict of interests regarding the publication of this article.

\section{References}

Bremaud, P., 1981. Markov Chains: Gibbs fields, Monte Carlo Simulation and Queues. 1st Edn., Springer Science and Business Media.
Carty, L.V. and J.S. Fons, 1994. Measuring changes in corporate credit quality. J. Fixed Income, 4: 27-41. DOI: $10.3905 /$ jfi.1994.408104

Christensen, J.H.E., E. Hansen and D. Lando, 2004. Confidence sets for continuous-time rating transition probabilities. J. Bank. Finance, 28: 2575-2602. DOI: 10.1016/j.jbankfin.2004.06.003

D'Amico, G. and P. Regnault, 2018. Dynamic measurement of poverty: Modeling and estimation. Sankhya B, 80: 305-340. DOI: $10.1007 / \mathrm{s} 13571-018-0153-6$

D'Amico, G., F. Petroni, P. Regnault, S. Scocchera and L. Storchi, 2019. A copula based Markov reward approach to the credit spread in European union. arXiv preprint arXiv:1902.00691.

D’Amico, G., G. Di Biase and R. Manca, 2012. Income inequality dynamic measurement of Markov models: Application to some European countries. Economic Modell., 29: 1598-1602.

D’Amico, G., G. Di Biase and R. Manca, 2014. Decomposition of the population dynamic Theil's entropy and its application to four European countries. Hitotsubashi J. Econom., 229-239.

D'Amico, G., G. Di Biase, J. Janssen and R. Manca, 2017. Semi-Markov Migration Models for Credit Risk. 1st Edn., John Wiley and Sons, ISBN-10: 9781848219052, pp: 316.

D’Amico, G., J. Janssen and R. Manca, 2006. Homogeneous semi-Markov reliability models for credit risk management. Dec. Econom. Finance, 28: 79-93. DOI: $10.1007 / \mathrm{s} 10203-005-0055-8$

D'Amico, G., J. Janssen and R. Manca, 2011. A nonhomogeneous semimarkov reward model for the credit spread computation. Int. J. Theoretical Applied Finance, 14: 221-238. DOI: $10.1142 / \mathrm{S} 0219024911006346$

D'Amico, G., S. Scocchera and L. Storchi, 2018a. Financial risk distribution in European union. Physica A: Stat, Mechan. Applic., 505: 252-267. DOI: 10.1016/j.physa.2018.03.069

D'Amico, G., P. Regnault, S. Scocchera and L. Storchi, 2018b. A continuous-time inequality measure applied to financial risk: The case of the European union. Int. J. Financial Stud., 6: 62-62. DOI: $10.3390 / \mathrm{ijfs} 6030062$

Do Van, P., A. Barros and C. Berenguer, 2010. From differential to difference importance measures for Markov reliability models. Eur. J. Operat. Res., 204: 513-521. DOI: 10.1016/j.ejor.2009.11.025

Doubleday, K.J. and J.N. Esunge, 2011. Application of Markov chains to stock trends. J. Math. Stat., 7: 103-106. DOI: 10.3844/jmssp.2011.103.106

Haviv, M. and L. Van der Heyden, 1984. Perturbation bounds for the stationary probabilities of a finite Markov chain. Adv. Applied Probability, 16: 804-818. DOI: $10.2307 / 1427341$ 
Huang, J.Z. and M. Huang, 2012. How much of the corporate-treasury yield spread is due to credit risk? Rev. Asset Pric. Stud., 2: 153-202. DOI: 10.1093/rapstu/ras011

Jarrow, R.A., D. Lando and S.M. Turnbull, 1997. A Markov model for the term structure of credit risk spreads. Rev. Financial Stud., 10: 481-523. DOI: $10.1093 / \mathrm{rfs} / 10.2 .481$

Lando, D. and T.M. Skødeberg, 2002. Analyzing rating transitions and rating drift with continuous observations. J. Bank. Finance, 26: 423-444. DOI: $10.1016 / \mathrm{S} 0378-4266(01) 00228-\mathrm{X}$

Ramesh, A.V. and K. Trivedi, 1993. On the sensitivity of transient solutions of Markov models. ACM SIGMETRICS Performance Evaluat. Rev., 21: 122-134. DOI: 10.1145/166962.166998

Sadek, A. and N. Limnios, 2005. Nonparametric estimation of reliability and survival function for continuous-time finite Markov processes. J. Stat. Plann. Inference, 133: 1-21.

DOI: $10.1016 /$ j.jspi.2004.03.010
Schweitzer, P.J., 1968. Perturbation theory and finite Markov chains. J. Applied Probability, 5: 401-413. DOI: $10.2307 / 3212261$

Shannon, C.E., 1948. A mathematical theory of communication. Bell Syst. Technical J., 27: 379-423. DOI: $10.1002 / \mathrm{j} .1538-7305.1948 . t b 01338 . \mathrm{x}$

Theil, H., 1967. Economics and information theory. Technical Report.

Thyagarajan, V. and M. Saiful, 2005. Retail banking loan portfolio equilibrium mix: A Markov chain model analysis. Am. J. Applied Sci., 2: 410-419. DOI: 10.3844/ajassp.2005.410.419

Trueck, S. and S.T. Rachev, 2009. Rating based Modeling of Credit Risk: Theory and Application of Migration Matrices. 1st Edn., Academic Press, Burlington, ISBN-10: 0080920306, pp: 280.

Yu, J.Y., S. Mannor and N. Shimkin, 2009. Markov decision processes with arbitrary reward processes. Math. Operat. Res., 34: 737-757.

DOI: $10.1287 /$ moor. 1090.0397 